\title{
Characterization of hospital-acquired pneumonia in Intensive Care Unit. General Hospital
}

\author{
Dixan Negreira Ochoa*, Ledianet Alvarez Infante, Ramon Boch, Eliades Millan Cordovi, Carlos Montero Pita \\ "Guillermo Dominguez Lopez", Las Tunas, Cuba.
}

*Corresponding author: Dixan Negreira Ochoa, "Guillermo Dominguez Lopez”, Las Tunas, Cuba.

Received date: March 10, 2020; Accepted date: April 28, 2020; Published date: May 12, 2020

Citation: Dixan Negreira Ochoa, Characterization of hospital-acquired pneumonia in Intensive Care Unit. General Hospital. J Clinical Research and Reports, , 4(1); DOI:10.31579/2690-1919/067

Copyright: () 2020 Dixan Negreira Ochoa, This is an open access article distributed under the Creative Commons Attribution License, which permits unrestricted use, distribution, and reproduction in any medium, provided the original work is properly cited.

\begin{abstract}
Objective: characterize the hospital-acquired pneumonia (HAN) in the Intensive Care Unit (ICU) of the

Methods: a descriptive and cross-sectional study was carried out to characterize the hospital-acquired pneumonia in admitted patient in ICU of the General Hospital "Guillermo Domínguez López" in Puerto Padre, Las Tunas since June, 2018 to May, 2019. The population was all the patients who acquired the infection during the admission. The information was taken from de patient's clinic file. It was created graphics and charts to pick the information. Dates was described, analyzed and compared with others national and international studies.
\end{abstract}

Result: the prevalent age group was $60-79$ to $59 \%$. Asisted mechanical ventilation was not realed with the HAN due to the procedure was only performing in 9 patients to $25 \%$. The most frequent isolated germ was citrobacter. The deseases which was most related with (HAN) was neurological deseases. The mortality was high, 20 patients die to $58 \%$.

Conclusion: clinic and epidemiologic characterisctics of NIH was described in the ICU of the General Hospital "Guillermo Domínguez López".

Key words: hospital-acquired pneumonia, mechanical ventilation, mortality, germs, antibiotics

\section{Introduction}

Durante siglos la humanidad se ha enfrentado a diversas problemáticas de salud, las enfermedades infecciosas que muchos atribuían a influencias sobrenaturales, en la edad de piedra, fueron de las que más azotaron al hombre hasta su posterior evolución intelectual (1).

Las infecciones respiratorias y en particular las neumonías, tan frecuentes en tiempos de guerra y catástrofes, tuvieron gran participación en la necesidad del hombre por buscar una solución. Se sabe que las muertes masivas, por contagio y ausencia de sustancias que pudieran ayudar a sanar al enfermo, eran abrumadoras. No fue hasta la era de los antibióticos que pudo tener un mayor conocimiento del tema (2).

Hoy en día el hombre no muere por una infección respiratoria, gracias a la gran variedad de antibióticos sintetizados, pero de forma inversa y contradictoria, se sabe que los servicios médicos tiene protagonismo en el aumento de la frecuencia de neumonías causadas por otros gérmenes diferentes a los que comúnmente se adquieren en la comunidad, las que se debe poner mayor énfasis y control con antibióticos de mayor potencia, las llamadas neumonías intrahospitalaria(NIH) o asociadas a los servicios de médicos(NASM)(3).
En países del primer mundo constituye una de las principales causas de sepsis intrahospitalarias, en España y México ocupan el tercer lugar,(2) mientras que en Estados Unidos solo fueron precedidas por la infecciones urinarias (3).

La mortalidad de la NN en estos pacientes asciende desde un $30 \%$ hasta un $70 \%$, aunque se debe tener en cuenta que este porcentaje depende de muchos factores y es muy difícil atribuir la mortalidad a la NN en concreto (4).

La mortalidad atribuible por neumonía asociada a ventilación mecánica (NAVM) se ha definido como el porcentaje de muertes que no hubieran ocurrido en ausencia de la infección. Numerosos estudios y controles de casos (5) han estimado que entre un $33 \%$ y un 50\% de todas las muertes relacionadas con la NAVM suceden como resultado directo de la infección, con una mortalidad más alta en pacientes infectados por Pseudomona aeruginosa o Acinetobacterspp y asociados a bacteriemia(6).

En Cuba se aprecia un comportamiento similar y ocupa el foco principal de sepsis en las salas de terapia intensiva y contribuye a aumentar las pérdidas humanas y elevar los costos hospitalarios (7-8).

Nuestro país no queda aislado de esta problemática, estudios revelan que la mortalidad haciende a más del 50\% de los pacientes con NIH (9). Se considera la misma una causa de muerte evitable dentro de las 
enfermedades transmisibles donde existen una serie de factores de riesgos que se pueden modificar en vida del paciente para una mejor atención (8).

En la provincia Las Tunas se determinó que de cada 10 pacientes ingresados en UCI, 6 se complicaban con NIH y 2 morían, cantidad significativa que demandaba una mayor atención (9).

En nuestro hospital la neumonía nosocomial en pacientes ingresados en la UCI es común, datos estadísticos muestran que en el año 2016 la mortalidad fue de más del $50 \%$ de los pacientes complicados por NIH, además de las incontables pérdidas materiales que le ocasionan al hospital y a la familia. Para nuestro servicio, una de las metas más importantes es disminuir las neumonías nosocomiales, ya que inciden en un gran porcentaje de morbilidad y mortalidad. Esto nos ha motivado a realizar un estudio con el objetivo de caracterizar la neumonía en la población crítica en el HG Guillermo Domínguez López, así como entenderla en términos de: grupo de edad mayor afectado, relación de la ventilación mecánica en la aparición de NIH, momento del diagnóstico, patrones microbianos de la institución y mortalidad por la afección.

\section{DISCUSIÓN Y ANÁLISIS DE LOS RESULTADO}

El total de episodios de NIH en UCI en el periodo estudiado fue de 34 pacientes como se observa en la tabla 1 . El grupo etario más afectado fue el de 70-79 años con 20 pacientes para un 59\% y el sexo más afectado fue el masculino con 21 pacientes para un $64 \%$.

Al calcular la media a partir de la fórmula anterior:

$$
\bar{X}=\frac{\sum(M c \times f A)}{n}
$$

$\bar{X}=\frac{2443}{34}=71,852 \ldots \approx 71,9$

Encontramos que la edad promedio de todos los pacientes infectados fue 71 años. Como se puede ver el valor medio está dentro del grupo de edad de los pacientes más afectados.

\begin{tabular}{|c|c|c|c|c|c|c|}
\hline \multirow[t]{2}{*}{ Grupos de edades (años) } & \multicolumn{2}{|c|}{ Masculino } & \multicolumn{2}{|c|}{ Femenino } & \multicolumn{2}{|c|}{ Total } \\
\hline & $\mathbf{N}$ & $\%$ & $\mathbf{N}$ & $\%$ & $\mathbf{N}$ & $\%$ \\
\hline $19-29$ & 0 & 0 & 0 & 0 & 0 & 0 \\
\hline $30-49$ & 2 & 7 & 0 & 0 & 2 & 6 \\
\hline $50-59$ & 1 & 3 & 0 & 0 & 1 & 3 \\
\hline $60-69$ & 4 & 15 & 2 & 7 & 6 & 17 \\
\hline $70-79$ & 11 & 29 & 9 & 24 & 20 & 59 \\
\hline $80-89$ & 2 & 7 & 2 & 7 & 4 & 12 \\
\hline 90 y más & 1 & 3 & 0 & 0 & 1 & 3 \\
\hline Total & 21 & 62 & 13 & 38 & 34 & 100 \\
\hline
\end{tabular}

Tabla 1. Distribución de casos según grupos de edades y sexo.

En el hospital Ernesto Guevara de Las Tunas encontraron que el grupo de edad más afectado fue el de más de 60 años con 71 pacientes para un $41 \%$, seguido por el de 50 a 59 años (10).

Según José Gundián Gozalez-Piñeira, en un estudio realizado en el hospital Hermanos Ameijeiras, obtuvo como resultado que el grupo de edad mayor a 50 años fue el que mas predominó (11), el cual se asemeja a nuestro resultado. En este mismo estudio se reporto un predominio del sexo femenino con un $58 \%$.

Por su parte Carmen Alicia San José Arribas en su tesis doctoral encontró un predominio del sexo masculino y pacientes mayores de 60 años (12).

Sin embargo en otro estudio realizado en el hospital provincial de Granma, se encontró cifra diferente, obteniéndose predominancia en menores de 60 años. En relación con el sexo, fue más frecuente en los masculinos; el 72,0\% de pacientes afectados eran hombres (13).

En opinión de este autor a mayor edad, el sistema inmunológico se deprime proporcionando susceptibilidad a infecciones graves, además de que la asociación con enfermedades crónicas es más probable. Se considera que las edades extremas son un factor de riesgo importante en la incidencia de estas infecciones.

Este investigador encontró diferencias con respecto a los resultados obtenidos por Yoniel Milanés González en el hospital provincial de Bayamo que reportó una mayor incidencia en menores de 60 años (13), considero que pudiera estar en relación con un predominio de ingresos de adultos jóvenes en relación con los politraumatismos y quirúrgicos complejos. 
En opinión de este autor el predominio del sexo masculino pudiera estar en relación con el tabaquismo el cual es un factor de riesgo importante en las enfermedades aterogenicas. Estas enfermedades, como se demuestra en este estudio, son una de las principales causas de ingresos en nuestra unidad.
En la tabla 2 se correlacionan el número las enfermedades por la que fueron ingresado los pacientes estudiados asociadas con mayor frecuencia al desarrollo de NIH, donde se encontró un predominio de las neurológicas seguidas por las cardiovasculares con 13 y 12 pacientes respectivamente(44 y $35 \%)$.

\begin{tabular}{|c|rc|}
\hline Enfermedades & Nro & \% \\
\hline Neurológicas & 13 & 44 \\
\hline Cardiovasculares & 12 & 35 \\
\hline Respiratorias & 5 & 9 \\
\hline Quirúrgicas & 2 & 6 \\
\hline Otras & 2 & 6 \\
\hline Total & 34 & 100 \\
\hline
\end{tabular}

Tabla 2. Distribución de casos según según enfermedad subyacente.

La presencia de coma o la alteración de los reflejos de las vías aéreas superiores, que suelen acompañar a estas enfermedades, se han señalado como factores de riesgo de importancia, sobre todo en la neumonía de comienzo precoz. Es indudable que el estado de salud previo al ingreso y la condición que provoca dicho ingreso, ejerce un rol importante en el desarrollo de la neumonía nosocomial ya que las alteraciones que provoca el propio proceso morboso puede alterar los mecanismo de defensa del huésped (14).

En la investigación de César León-Chahua y col. reportaron como principales causas relacionadas a las NIH, las enfermedades neurológicas, alcanzando el $26 \%$ de la población estudiada (15).

Por su parte Liudmila Carnesoltas y col, encontraron una relación de enfermedades neurológicas con las NIH en 21 pacientes de la población estudiada para un $34.4 \%$ (16).

Sin embargo en un estudio realizado en el hospital provincial de Pinar del Rio estuvo e primer lugar las enfermedades respiratoria como causa principal de ingreso e pacientes que luego presentaron la complicación. Le seguía en segundo lugar las enfermedades cardiovasculares (17).
Es criterio del autor que las enfermedades neurológicas de forma general toman el primer lugar, y más particularmente las enfermedades cerebrovasculares, seguida por las enfermedades cardiovasculares, resultados que se asemejan a otros estudios. La alta incidencia de estas afecciones en la población de Puerto Padre contribuye a un aumento de pacientes con la necesidad de admisión en UCI. Existe un alto riesgo para los pacientes que ingresaron por estas afecciones presentar NN en los primeros días del ingreso. Es de vital importancia que el personal médico y de enfermería tome esto como referencia para prestar más atención a los signos que pudieran aparecer y que sugirieran la presentación de la infección. Además de tener presente las medidas que debe ser tomada en estos servicios con el objetivo de evitar la aparición de infecciones respiratorias como son el aspirado frecuente de secreciones bronquiales en pacientes entubados y la movilización del enfermo de la cama a la silla lo antes posible.

En la tabla \#3 se muestra la distribución de pacientes según momento del diagnostico, se encontró que 25 pacientes fueron diagnosticados entre dos y cuatro días y nueve pacientes a los cinco días o más para un $73.5 \%$ y un $26.6 \%$ respectivamente

\begin{tabular}{|c|cc|}
\hline $\begin{array}{c}\text { Momento del diagnostico } \\
\text { (días) }\end{array}$ & Nro. \\
\hline $2-4$ & 25 & 73.5 \\
\hline$\geq 5$ & 9 & 26.5 \\
\hline Total & 34 & 100 \\
\hline
\end{tabular}

Tabla 3. Distribución de casos según momento del diagnostico.

La NN se desarrolla en pacientes hospitalizados luego de 48 a 72 horas de la admisión o 7 días después del egreso (17).

Según Langer y col. en un estudio multicéntrico en unidades de terapia intensiva, fueron los primeros en clasificar la NN de aparición precoz (desarrollada en los primeros 4 días tras la hospitalización) y de aparición tardía (tras 5 días o más) (18). Sin embargo, no existen estudios diseñados de manera apropiada que avalen estos umbrales de tiempo.

De acuerdo con la American Thoracic Society, a partir del día cinco de hospitalización la flora nosocomial sustituye la flora bacteriana normal de los pacientes; siguiendo ese criterio, el día cinco se establece como punto de separación entre una y otra (19).
Según Cortinas Saenz y col, reportaron que en la neumonía precoz los patógenos más frecuentemente aislados fueron Staphylococcus aureus, en los casos de neumonía tardías resalta el género Pseudomonas (20).

La American Thoracic Society publicó unas guías para el diagnóstico y tratamiento de pacientes adultos con $\mathrm{NN}$, en las que se considera que los dos principales factores que determinan el tipo de antibióticos que se suministrará son el tiempo que lleva el paciente ingresado en el hospital, que permite clasificar la neumonía en precoz ( $<5$ días) o tardía ( $\geq 5$ días), y la presencia de factores de riesgo para infección por microorganismos potencialmente multirresistentes (21). 
Para el autor es de gran importancia que el personal médico encargado de actuar frente a esta complicación sea capaz de definir, clasificar la infección de acuerdo a estos lapsos de tiempo y proporcionar una terapéutica empírica de acuerdo con los gérmenes más frecuentes de acuerdo a los días de realizado el diagnostico. Esto, además de alertarnos acerca de la rápida instalación de la infección, nos ayuda a comprender que no debemos pasar por alto cualquier síntoma que nos oriente para llegar a un diagnostico precoz y proporcionar una terapéutica inmediata evitando la aparición de otras complicaciones o el estadio prolongado.

En la tabla 4 se relaciona la infección nosocomial con la ventilación mecánica, se encontró que 25 pacientes para un $75 \%$ no estaban asociados a dicho proceder, mientras que 9 pacientes para un $25 \%$ si se relacionaron.

\begin{tabular}{|c|cc|}
\hline Ventilación mecánica & Nro. $\%$ \\
\hline No & 25 & 75 \\
\hline $\mathrm{Si}$ & 9 & 25 \\
\hline Total & 34 & 100 \\
\hline
\end{tabular}

Tabla 4. Distribución de casos según ventilación mecánica.

Según Pérez Estrada y col. en estudio realizado en el año 2014 sobre infecciones nosocomiales en UCI reportaron una incidencia de neumonía nosocomial en ventilados del $43.8 \%$ (34) cifra que no se asemejan a la nuestra.

En su publicación sobre incidencia de los factores de riesgo de NAVM en pacientes críticos Cook y col (22), ha estimado que el riesgo de $\mathrm{NN}$ es de un $1 \%$ cada día de VM y que ese riesgo varía en función del tiempo, siendo del 3\% los 5 primeros días de VM, del 2\% del $5^{\circ}$ al $10^{\circ}$ día, y del $1 \%$ el resto de los días.

Por su parte Torres y col. plantearon que la mayoría de pacientes ventilados mecánicamente están con intubación orotraqueal menos de una semana, cerca de la mitad de los casos de NAVM ocurren durante los primeros días. La incidencia de la NAVM es del 10-30\% en pacientes que requieren VM más de 48 horas. Esta incidencia depende del tipo de población estudiada, de la presencia o ausencia de factores de riesgo y del tipo e intensidad de las medidas preventivas adoptadas (23).
En otra investigación realizada por Trouillet y colaboradores (24), informaron que a partir del séptimo día de ventilación mecánica, la posibilidad de infección por patógenos potencialmente multirresistentes aumenta considerablemente.

Es criterio de este autor que en este servicio la asociación de la asistencia ventilatoria con la aparición de NN está por debajo a lo reportado por otros autores $(23,24,34)$. Esto pudiera estar relacionado a que la necesidad de ventilación mecánica en los pacientes ingresados en el servicio es pobre y de practicarla no se extiende por largo periodo de tiempo, además de las estrictas medidas de sanidad y asepsia que se toman a la hora de realizar el proceder. Es importante tener en cuenta, por parte del personal de enfermería, el cumplimiento de normas y cuidados de la ventilación, además del reconocimiento y actuación sobre los factores de riesgo.

Al analizar la distribución de casos según realización de cultivos (tabla \#5), se encontró que fueron realizados en 31 pacientes para un $90 \%$, sin embargo a un $10 \%$ de los pacientes no se les realizó por cuestiones de déficit de materiales y medios de cultivos necesarios para la identificación del germen.

\begin{tabular}{|c|cc|}
\hline Cultivos & Nro. & $\%$ \\
\hline $\mathrm{Si}$ & 31 & 90 \\
\hline No & 3 & 10 \\
\hline Total & 34 & 100 \\
\hline
\end{tabular}

Tabla 5. Distribución de casos según realización de cultivos de secreciones respiratorias.

Según Trouillet JL y col. la presencia de bacterias en la vía aérea inferior de los pacientes intubados no es suficiente para diagnosticar una infección pulmonar verdadera. El árbol traqueo-bronquial y la orofaringe de los pacientes ventilados mecánicamente se encuentran frecuentemente colonizados por bacilos entéricos Gramnegativos (25).
En un estudio realizado por Beatriz W. y col. en el hospital José de San Martin de Buenos Aires informaron la realización 430 cultivos de secreciones aislándose gérmenes en la totalidad de las muestras tomadas (26).

En el artículo de Emili Díaz y col. recomienda obtener muestras de las secreciones pulmonares para confirmar el diagnóstico e identificar el patógeno responsable de la infección y así poder 
adecuar posteriormente el tratamiento antibiótico que se ha iniciado de forma empírica (36).

El autor considera que la importancia radica en la identificación por antibiograma del posible antibiótico a usar en cada caso. La resistencia de las bacterias a los antibióticos de amplio espectro exige la toma de muestras obligada, con el objetivo de aplicar una terapéutica lo más especifica posible, teniendo en cuenta que cada paciente no responde de la misma manera y que los antibiogramas son métodos in vitro con un pequeño porciento de inefectividad.
La utilización de métodos de cultivos, además de confirmar la infección, ayuda a identificar el germen causal y el antibiótico de elección. La no realización de estos métodos no está justificada, ya sea esputos, aspirado traqueal, hemocultivos, etc.

Como se observa en la tabla 6 encontramos que el germen más frecuente fue el citrobacter, aislado en 10 casos, seguido por enterobacter y estafilococo en cinco casos cada uno para un 33 y 17 $\%$ respectivamente. Además de la alta frecuencia, también observamos que de los 10 cultivos en donde se aisló el citrobacter en 7 presentaba resistencia.

\begin{tabular}{|c|c|c|c|c|}
\hline \multirow[t]{2}{*}{ Germen aislado } & \multicolumn{2}{|c|}{ Resultado del antibiograma } & \multicolumn{2}{|c|}{ Total } \\
\hline & Resistencia & Sensibilidad & No & $\%$ \\
\hline Citrobater & 7 & 3 & 10 & 33 \\
\hline Enterobacter & 2 & 3 & 5 & 17 \\
\hline Estafilococo ssp & 0 & 5 & 5 & 17 \\
\hline Pseudomona & 0 & 4 & 4 & 12 \\
\hline Proteus & 0 & 4 & 4 & 12 \\
\hline Klepsiella & 0 & 2 & 2 & 6 \\
\hline Basilo no fermentador & 0 & 1 & 1 & 3 \\
\hline Total & 9 & 22 & 31 & 100 \\
\hline
\end{tabular}

Según Chastre (27), los microorganismos aislados de manera más frecuente en las secreciones bronquiales de los pacientes con NN son S. aureus y P. aeruginosa, que suponen el $50 \%$ de los microorganismos aislados. A estos les siguen, por orden de frecuencia, los siguientes: Enterobacterias (E. coli, Klebsiella spp., Enterobacter spp., Citrobacter spp., Serratia spp., y Proteus spp.) presentes en el 15\%, bacilos Gramnegativos no fermentadores distintos de la P. aeruginosa (Acinetobacter spp., Stenotrophomonas spp., y Burkholderia spp.) en el $10 \%$, y $\mathrm{H}$. influenzae y $\mathrm{S}$. pneumoniae (entre otros) en el resto de los casos.

Según Danilo Eranio y col. en su estudio realizado en el hospital Ernesto Guevara reportaron que el germen más frecuente fue el bacilo no fermentador aislándose en el $76 \%$ de los cultivos de secreciones respiratorias (22).
Por otro lado José Gundián Gozalez-Piñeira reportan en su estudio, una mayor incidencia de psudomona como microorganismo causante de NN en UCI (23).

Por su parte Domínguez Pereira informa que los gérmenes más frecuente en pacientes con NN en el hospital Arnaldo Milian Castro fueron acinetobacter seguido por estafilococos, psudomona y el citrobacter (27)

$\mathrm{El}$ autor considera que nuestro resultado de forma general coincide con lo reportado por la literatura que informa mayor frecuencia de los gram negativos. Otros patógenos que le siguen en mayor frecuencia es el estafilococo. Siempre debemos tener en cuenta que los gérmenes responsables de NIH varían de acuerdo con la duración de la ventilación mecánica, patrones locales de distribución, la existencia o no de tratamiento antibiótico previo, enfermedades asociadas en el paciente y la estadía hospitalaria. 
Gráfico 1.

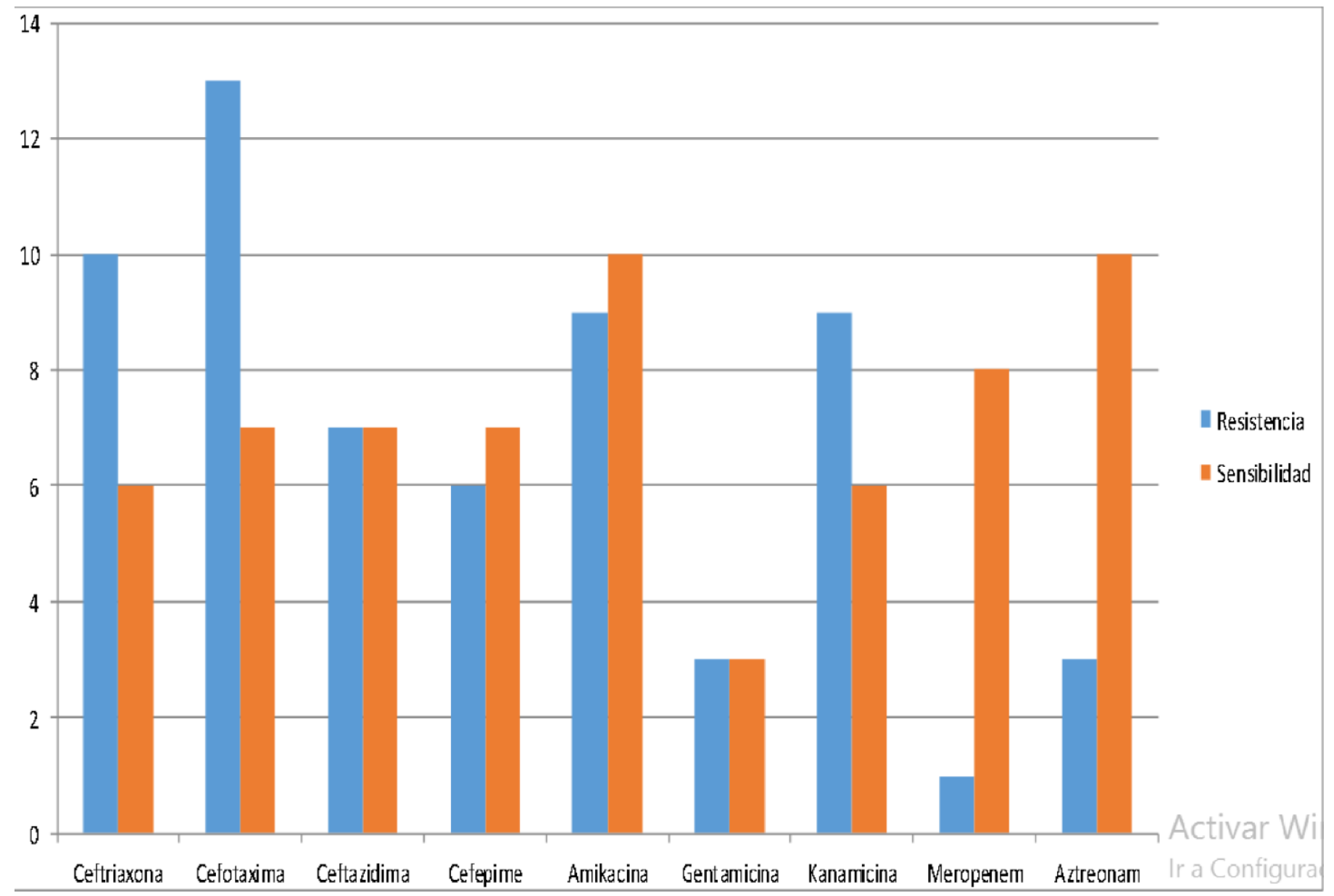

En el gráfico 1 se muestran los antibióticos utilizados in vitro para determinar sensibilidad y resistencia de los gérmenes según cultivo, en el eje de las $\mathrm{X}$ se muestran los principales antibióticos y en el de las $\mathrm{Y}$ el número de cultivos.

Según Belén Arasil-Garcia y col, en su estudio realizado en el centro nacional de microbiología de Madrid, informan que la resistencia a las cefalosporinas de 3ra generación a aumentado de 9,8\% en el 2010 a un $19 \%$ en el 2014 (28).

Por otro lado Sarubi F y col reportan una mayor eficacia del meropenem frente a las NN causadas por gérmenes resistentes a la amikacina y cefalosporinas de tercera generación (29).

En su artículo, José Blanquer considera que en pacientes con NIH de inicio precoz y sin factores de riesgo para multiresistencia se deben cubrir patógenos que son generalmente de origen comunitario y con baja probabilidad de multirresistencias. Por el contrario, los pacientes con NIH de origen tardío o con presencia de factores de riesgo para multiresistencia deben recibir tratamiento empírico inicial de amplio espectro, y en combinación, para garantizar la cobertura de la mayoría de microorganismos causales en este grupo de enfermos (30).
El autor considera que un dato interesante es la aparición de resistencia a la cefotaxima en 13 de los cultivos realizados y de la alta sensibilidad de los gérmenes aislados al meropenem y a la amikacina. La resistencia antibiótica es uno de los temas más polémicos en nuestros días, en nuestro medio se ha podido comprobar que la necesidad de sustancias más eficaces, es evidente. En varia ocasiones, nuestro hospital no pudo contar con antibióticos de amplio espectro como el aztreonam o la meropenem por lo que el tratamiento adecuado a estos pacientes no fue el más eficaz.

Si bien es importante el diagnóstico precoz, lo es más la disponibilidad antibiótica que se necesita para combatir la infección presente, la cual, por causas ajenas al personal médico, se ha visto afectada en varias ocasiones. La utilización de antibióticos de amplio espectro de forma empírica antes del resultado de los cultivos está justificada, sin embargo, en nuestro medio no siempre están disponibles estos medicamentos tan necesarios.

En el gráfico 2 se muestran que el número de pacientes fallecidos por causa de la infección fueron de 20 casos para un 59\%. 


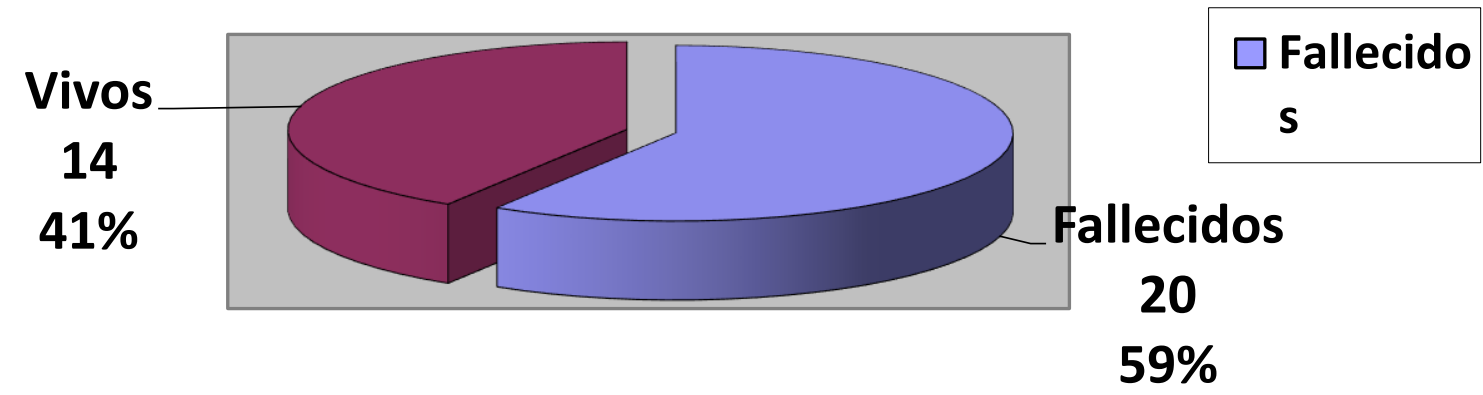

Gráfico 2. Distribución de casos según mortalidad.

Según Valles J. la mortalidad de la NIH en estos pacientes asciende desde un $30 \%$ hasta un $70 \%$, aunque se debe tener en cuenta que este porcentaje depende de muchos factores y es muy difícil atribuir la mortalidad a la NN en concreto (31).

Por su parte, en un estudio multicéntrico realizado por Lager $\mathrm{M}$ y col. reportan que entre un $33 \%$ y un $50 \%$ de todas las muertes relacionadas con la NN suceden como resultado directo de la infección (32).

Por otro lado Martha Abascal en su estudio encontró una mortalidad del $64 \%$ de los pacientes diagnosticados de NIHen el hospital militar Manuel Fajardo (33).

El autor considera que la mortalidad a causa de la NIH en otros estudios y la encontrada en esta investigación, además coincidir, son alarmantes. Constituye un problema en el cual debemos prestar más interés por la pérdida de vidas humanas, por un motivo diferente al que se admitió en el servicio. Las probabilidades de muerte luego de adquirida la infección ascienden por encima de un $50 \%$, dato que, además de alertarnos sobre la gravedad de la afección, nos obliga a tomar medidas para la resolución lo más temprano posible utilizando todo los medios disponibles a nuestro alcance.

\section{CONCLUSIONES}

Durante el periodo estudiado se determinó que el grupo de edad en que más frecuentemente se produce las NIH es el de 70 a 79 años. La aparición de los primeros síntomas de la infección generalmente es en los primeros días. La asociación de la ventilación mecánica con el desarrollo de la enfermedad no es semejante a otros estudios.

Se le indicaron cultivo al $90 \%$ de los pacientes, quedando un $10 \%$ sin un tratamiento específico, debido al déficit de materiales necesarios para su realización. El citrobacter fue el microorganismo etológico responsable del desarrollo de la mayoría de las NIH en el periodo estudio, además de poseer una gran resistencia antibiótica.

Uno de los antibióticos que se demostró ha perdido efectividad frente a las bacterias aisladas es la cefotaxima mientras que otros como el meropenem continua demostrando su espectro antimicrobiano frente a estos patógenos. Las afecciones que llevaron al paciente a ingresar en nuestro servicio y que más se asociaron al desarrollo de la infección fueron las enfermedades neurológicas. La mortalidad es alta, llegando alcanzar más del 50\% de los pacientes aquejados de la infección.

\section{References}

1. Pérez Rojo R, Moreno Zabaleta R, Álvarez González CJ. Neumonía Nosocomial. En Patología Respiratoria. Manual de Actuación. Madrid 2014.Capítulo 27. Pags 189-199.

2. Morales Suárez M, Varela M. Estudio comparativo de prevalencia de infecciones intrahospitalaria en un hospital comunal valenciano. Rev. Enfermedades Infecciosas.2015;7(13):23-32.

3. Ronald N.J. Impact of changing pathogens and antimicrobial susceptibility patterns in the treatment of serious infections in hospitalized patients. Am $\mathrm{J}$ Med 2006;100(6):3S-12S.

4. Valles J, Pobo A, Garcia-Esquirol O, Mariscal D, Real J, Fernandez R. Excess ICU mortality attributable to ventilator-associated pneumonia: the role of early vs late onset. IntensiveCareMed 2012 Aug;33(8):1363-8.

5. Hernández Ávila M. Epidemiología: diseño y análisis de estudios. Ed. Panamericana; 2007.

6. Bonten M J, Kollef MH, Hall JB. Risk factors for ventilator-associated pneumonia: from epidemiology to patient management. Clin Infect Dis 2014 Apr 15;38:11419.

7. Soler Rodriguez M, Soneira Pérez J, Fragoso López R, Ribero López JC, Pérez Delgado S. Cuidados Intensivos. RevCubanaMed. 2013;32(2):77-84.

8. Prat A, Asenjo MA, Las infecciones nosocomiales como indicador de calidad de la asistencia hospitalaria. Repercusión económica de las infecciones nosocomiales.

9. Utra Cardoso Danilo Eranio.Caracterización de pacientes con bronconeumonía bacteriana asociada a la ventilación artificial mecánica.Revista Electrónica Dr. Zoilo E. Marinello Vidaurreta Vol. 40, número 8 ISSN 1029-3027| RNPS 1824 agosto 2015.

10. Danilo Eranio Utra Cardoso. Caracterización de pacientes con bronconeumonía bacteriana asociada a la ventilación artificial mecánica. Revista Electrónica Dr. Zoilo E. Marinello Vidaurreta Vol. 40, número 8ISSN 1029- 3027 | RNPS 1824 agosto 2015.

11. José Gundián Gozalez-Piñeira. Neumonía Nosocomial. Resultado de un año de trabajo en la UCI del Hospital Hermanos Ameijeiras. Revista Cubana de Medicina Intensiva $\mathrm{y}$ Emergencias Rev Cub Med Int Emerg 2002;1(32-41).

12. Carmen Alicia San José Arribas. NEUMONÍA EN LA UCI ASOCIADA O NO A LA VENTILACIÓN MECÁNICA: CARACTERÍSTICAS Y FACTORES DE 
MORTALIDAD HOSPITALARIA. Tesis Doctoral. Universidad de Valladolid.0

13. Oniel Milanés González. Caracterización clínico epidemiológica de la neumonía asociada a la ventilación mecánica artificial. hospital provincial universitario Carlos Manuel de Céspedes desde enero-diciembre de 2010. Bayamo, Granma.

14. M. A. Domingues Perez. A. Caballero. Neumonia Nosocomial. Cap 101 Terapia Intesiva tomo IV. La Habana. Editorial Ciencias Médicas 2009.

15. César León-Chahua, Teodoro Oscanoa-Espinoza, Cynthia Chávez-Gutiérrez, José Chávez-Gutiérrez. Características epidemiológicas de la neumonía intr a hospitalaria en un servicio de medicina intensiva del Hospital Guillermo Almenara Irigoyen de Lima, Perú. Horiz Med 2016; 16 (3): 43-49.

16. Liudmila Carnesoltas Suarez, Miguel Ángel Serra Valdés, Rosario O'Farrill Lazo. Factores de riesgo y mortalidad por neumonía intrahospitalaria en la Unidad de Terapia Intensiva. Medwave 2013 Mar;13(2):e5637 doi: 10.5867/medwave.2013.02.5637.

17. Liliana Barcón Díaz, Reinier González Rodríguez. La neumonía nosocomial en hospital provincial de Pinar del Río. Rev. Ciencias Médicas. Marzo-abril, 2019; 23(2): 187194 ISSN: 1561-3194.

18. Langer M, Cigada M, Mandelli M, Mosconi P, Tognoni G. Early-onset pneumonia: a multicenter study in intensive care units. IntensiveCareMed1987;13:342-6.

19. American Thoracic Society. Hospital-acquired pneumonia in adults, diagnosis, assessment, initial severity and prevention. A consensusstatement. Am J RespirCritCareMed1996;153:1711-25.

20. Cortinas Saenz M, Lizan Garcia M, Jimenez-Visuete JM, Moreno Cuesta J, Cuesta Garcia J, Peyro Garcia R, Incidencia de las neumonías nosocomiales precoces $\mathrm{y}$ tardías asociadas a ventilación mecánica en una unidad de reanimación-críticos polivalente. Revista Española de Anestesiología y Reanimación 2007;54(3): 147-154.

21. ATS Guidelines for the Management of adults with hospital-acquired ventilator-associated, and healthcareassociated pneumonia. Am J Respir Crit Care Med 2005; 171:388-416.

22. Cook DJ, Walter SD, Cook RJ, Griffith LE, Guyatt GH, Leasa D, et al. Incidence of and risk factors for ventilatorassociated pneumonia in critically ill patients. Ann Intern Med 1998 Sep 15;129:433-40.

23. Torres A, Ferrer M, Badía J. Treatment guidelines and outcomes of hospital-adquired and ventilator-associated pneumonia. ClinicalInfectiousDiseases 2010;51(Suppl1):S 48-S 53.

24. Trouillet JL, Chastre J, Vugnat A, et al. Ventilatorassociated pneumonia caused by potentially drug-resistant bacteria. Am J Respir Crit Care Med 1998;157:531-9.
25. American Thoracic Society. Guidelines for the Management of Adults with Hospital-acquired, Ventilatorassociated, and Healthcare-associated Pneumonia. Am J RespirCritCareMed 2005 Feb 15;171:388-416.

26. BEATRIZ WEYLAND*, BEATRIZ PERAZZI, SUSANA GARCIA, CARLOS RODRÍGUEZ, CARLOS VAY, ANGELA FAMIGLIETTI. Etiología bacteriana de la neumonía nosocomial y resistencia a los antimicrobianos en pacientes con y sin tratamiento antimicrobiano previo. 011) 43: 18-23 ISSN 0325-7541 Revista Argentina de Microbiología (2011) 43: 18-23.

27. Chastre J, Trouillet JL, Vuagnat A, Joly-Guillou ML, Clavier H, Dombret MC, Gibert C. Nosocomial pneumonia in patients with acute respiratory distress syndrome. Am J RespirCritCareMed 1998; 157: 1165-72.

28. Belen Aracil-Garcia, Jesus Oteo Iglesias, Oscar Cuevas Lobato. Rapido aumento de la resistencia a cefalosporinas de $3^{\text {a }}$ generación, imipenem y de la corticoresistencia en 7.140 aislados de Klepsiella p. en hemocultivos (20102014) según datos de EARS-Net en Espana. revenfinfymicrob, vol 35 num 8 .

29. Sarubi F, Fransus B \& Verghese. Comparative activity of meropenem against major respiratory pathogens and amikacin-resistant nosocomial isolates.Eur $\mathrm{J}$ Clin Microbiol Infect Dis 2019; $11: 65-8$.

30. José Blanquer, Javier Aspa, Antonio Anzueto, Miguel Ferrer, Miguel Gallego, Olga Rajas, Jordi Rello, Felipe Rodríguez de Castro y Antonio Torres. Normativa SEPAR: neumonía nosocomial. Arch Bronconeumol. 2011;47(10):510-520.

31. Valles J, Pobo A, Garcia-Esquirol O, Mariscal D, Real J, Fernandez R. Excess ICU mortality attributable to ventilator-associated pneumonia: the role of early vs late onset. IntensiveCareMed 2007 Aug;33(8):1363-8.

32. Langer M, Cigada M, Mandelli M, Mosconi P, Tognoni G. Early-onset pneumonia: a multicenter study in intensive care units. IntensiveCareMed1987;13:342-6.

33. Marta Abascal Cabrera, Rafael González Rubio, Alberto de la Rosa Domínguez, Félix Ulloa Quintana. Repercusión de la bronconeumonía en la mortalidad hospitalaria. Rev Cubana Med Milit. 2001;30(2):99-105.

34. Pérez Estrada FA, Martínez Rodríguez I, Rojas Borroto CA, Mato Hernández M, Pérez Román E. Infección nosocomial en unidades de cuidados intensivos. Rev. Cubana MedIntEmerg [Internet]. 2014 [citado Nov 25, 2017]; 13(2):116-25.

35. Emili Díaz, Ignacio Martín-Loeches y Jordi Vallés. Formación médica continuada: Infección nosocomial. Fundamentos y actuación clínica. Neumonía nosocomial. EnfermInfeccMicrobiol Clin. 2013;31(10):692-698. 
Ready to submit your research? Choose Auctores and benefit from:

This work is licensed under Creative

Commons Attribution 4.0 License

\section{To Submit Your Article Click Here: Submit Article}

DOI:10.31579/2690-1919/067

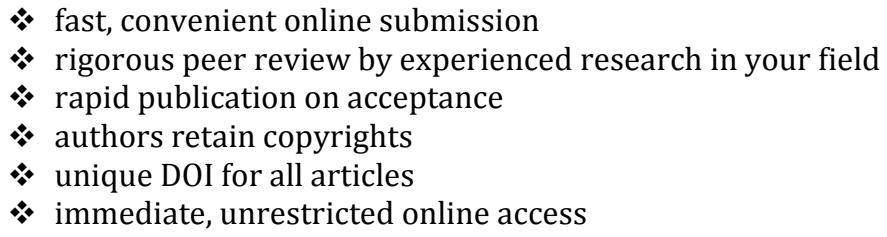

At Auctores, research is always in progress.

Learn more https://www.auctoresonline.org/journals/journal-of-clinicalresearch-and-reports 